\title{
Transcription as a tool for increasing metalinguistic awareness in learners of German Sign Language as a second language
}

\author{
Emily Kaufmann*, Reiner Griebel, Thomas Kaul \\ University of Cologne, Faculty of Human Sciences, Education \\ and Rehabilitation of the Deaf and Hard of Hearing
}

\begin{abstract}
Especially in adult second language learners, metalinguistic awareness is an important component in acquiring language competence. This paper reports on a study which evaluates a new transcription-based language training technique designed to improve sign language L2 learners' metalinguistic awareness, focusing on the area of non-manual features (mouth gestures and eyebrow activation), an area which typically poses great difficulty for hearing adult sign language learners. This study focuses on idiomatic signs (e.g. f-hand-to-forehead, meaning 'no idea') and questions because they contain mouth gestures and eyebrow activation. Participants $(\mathrm{N}=33)$ were hearing native speakers of German and students in the Deaf Education program at the University of Cologne in a fifth-semester German Sign Language (DGS) course during the study period. The study was conducted exclusively during the regular class time in the DGS 5 classes. The study was designed as follows. There were five training sessions, in each of which students were shown one video of a DGS sentence containing an idiomatic sign. The video was replayed as desired by participants, and the sentence and the idiomatic sign were discussed. In Group 1 $(\mathrm{N}=18)$, the control group, this standard approach for teaching idiomatic signs was used. In Group $2(\mathrm{~N}=15)$, the test group, the
\end{abstract}

transcription task was used: the idiomatic sign was discussed as above, and participants were additionally asked to transcribe the sentences, providing a gloss, mouth gestures, mouthings, and eyebrow activation. Following the five training sessions was the testing session, which was the same for both groups. Participants watched 12 videos, each of which presented one complete DGS sentence which contained either a mouth gesture or eyebrow activation as a salient feature. Half of the videos of contained an error in the respective non-manual feature and half were error-free. For each item, participants were instructed to decide whether or not the sentence contained an error and if so, state how to correct it. As in previous studies, the ability to detect and correct errors is used as a measure of metalinguistic awareness. Performance was compared between the two groups, and results show a significant difference between the groups $(p<.01)$, with the test group performing better on the error detection/correction task. This result indicates that DGS learners' metalinguistic awareness and by extension, their competence, in the area of non-manuals can be improved by integrating transcription techniques into DGS courses. Future studies on other techniques using video technology may prove equally promising.

\footnotetext{
*emily.kaufmann@uni-koeln.de
} 